\title{
ON COMPLEX QUADRATIC FIELDS WITH CLASS NUMBER EQUAL TO ONE( ${ }^{(1)}$
}

\author{
BY \\ HAROLD STARK
}

Let $R(\sqrt{ }-p)$ be a quadratic extension of the rationals, where $p$ is a positive square free integer. For nine values of $p$, namely $1,2,3,7,11,19,43,67,163$, the integers of $R(\sqrt{ }-p)$ form a unique factorization domain. Heilbronn and Linfoot [1] have shown that there is at most one more such value of $p$, and Lehmer [2] has shown that $p$ must be a prime greater than $5 \cdot 10^{9}$. In the present paper we verify and extend the lower bound of $5 \cdot 10^{9}$ for $p$. The result is

THEOREM 1. If the ring of integers of $R(\sqrt{ }-p)$ (p square free) forms a unique factorization domain, and $p>10^{4}$, then $p>\exp \left(2.2 \cdot 10^{7}\right)$.

It will be assumed throughout that $p$ is an integer satisfying the hypothesis of Theorem 1. We start with a formula equivalent to that given by Lemma 2 of [1].

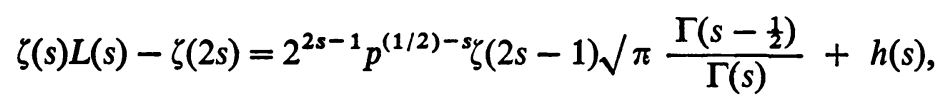

valid for $\sigma>\frac{1}{2}$, where $\zeta(s)$ is the Riemann zeta function, $L(s)$ is the Dirichlet $L$-series formed with the quadratic character $(\bmod p)$, and

$$
h(s)=\sum_{j=1}^{\infty} \int_{-\infty}^{\infty}\left(x-[x]-\frac{1}{2}\right) \frac{d}{d x}\left\{\left[\left(x+\frac{j}{2}\right)^{2}+\frac{p j^{2}}{4}\right]^{-s}\right\} d x .
$$

Let $x+(j / 2)=u(j \sqrt{ } p) / 2$ give a change of variable from $x$ to $u$ and integrate by parts $2 m-1$ times; we get, for $m=1,2, \cdots$,

(2) $h(s)=-\sum_{j=1}^{\infty}\left(\frac{2}{j \sqrt{ } p}\right)^{2 m+2 s-1} \int_{-\infty}^{\infty} \frac{B_{2 m}(x-[x])}{(2 m) !} \frac{d^{(2 m)}}{d u^{(2 m)}}\left\{\left(u^{2}+1\right)^{-s}\right\} d u$,

where $B_{k}(x)$ is the $k$ th Bernoulli polynomial $\left(B_{0}(x)=1, B_{1}(x)=x-\frac{1}{2}\right.$, $\left.B_{2}(x)=x^{2}-x+\frac{1}{6}, \cdots\right)$ and the series converges for $\operatorname{Re} s>1-m$.

It is well known ([3, p. 245], Jordan's $\left.\varphi_{n}(x)=B_{n}(x) / n !\right)$ that for $0 \leqq x \leqq 1, k>1$,

Received by the editors May 6, 1965.

(1) This paper represents a portion of my Ph. D. dissertation written under the supervision of Professor D. H. Lehmer. 
(3)

$$
\left|\frac{B_{k}(x)}{(k) !}\right| \leqq \frac{2}{(2 \pi)^{k}} \zeta(k),
$$

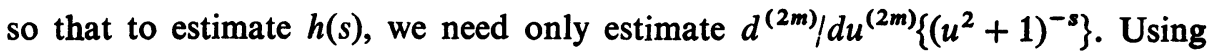
the fact that

$$
\frac{d^{2}}{d u^{2}}\left\{\left(u^{2}+1\right)^{-s}\right\}=2 s(2 s+1)\left(u^{2}+1\right)^{-s-1}-2 s(2 s+2)\left(u^{2}+1\right)^{-s-2},
$$

we see inductively that we can write

$$
\frac{d^{(2 m)}}{d u^{(2 m)}}\left\{\left(u^{2}+1\right)^{-s}\right\}=\sum_{k=0}^{m} c_{m k}(s)\left(u^{2}+1\right)^{-s-m-k} .
$$

Again, by induction we have

$$
\left|c_{m k}(s)\right| \leqq\left(\begin{array}{c}
m \\
k
\end{array}\right) \prod_{j=0}^{2 m-1}(2|s|+2 j) .
$$

Thus

$$
\begin{aligned}
\left|\frac{d^{(2 m)}}{d u^{2(m)}}\left\{\left(u^{2}+1\right)^{-s}\right\}\right| & \leqq \sum_{k=0}^{m}\left(\begin{array}{c}
m \\
k
\end{array}\right)\left(u^{2}+1\right)^{-\sigma-m-k} \prod_{j=0}^{2 m-1}(2|s|+2 j) \\
& \leqq 2^{m}\left(u^{2}+1\right)^{-\sigma-m} \prod_{j=0}^{m-1}(2|s|+2 j)(2|s| \\
& \leqq 2^{m}\left(u^{2}+1\right)^{-\sigma-m}(2|s|+2 m-1)^{2 m} .
\end{aligned}
$$

In view of (2), (3) and (6), we have for $\sigma \geqq \frac{1}{2}$ and $m \geqq 1$ :

$|h(s)|$

$$
\begin{aligned}
& \leqq \sum_{y=1}^{\infty}\left(\frac{2}{y \sqrt{ } p}\right)^{2 m+2 \sigma-1} \cdot \frac{2}{(2 \pi)^{2 m}} \zeta(2 m) \cdot 2^{m}(2|s|+2 m-1)^{2 m} \int_{-\infty}^{\infty}\left(u^{2}+1\right)^{-1} d u \\
& \leqq 2 \pi \zeta^{2}(2 m)\left(\frac{4|s|+4 m-2}{\pi \sqrt{ }(2 p)}\right)^{2 m}
\end{aligned}
$$

(7)

$$
<2 \pi\left(\frac{2 m}{2 m-1}\right)^{2}\left(\frac{4|s|+4 m-2}{\pi \sqrt{ }(2 p)}\right)^{2 m} \text {. }
$$

Letting $m=30$, we see that if $|s| \leqq 22$ and $\sigma \geqq \frac{1}{2}$ (and of course $p>10,000$ ), then

$$
|h(s)|<10^{-19} \text {. }
$$

Let $\theta$ denote a number, complex or real, not necessarily the same each time it occurs, which satisfies $|\theta| \leqq 1$. We find that for $|s|<22$ and $\sigma \geqq \frac{1}{2}$, (1) becomes 


$$
\zeta(s) L(s)-\zeta(2 s)=\zeta(2-2 s) \frac{\Gamma(1-s)}{\Gamma(s)}\left(\frac{\sqrt{ } p}{2 \pi}\right)^{1-2 s}+10^{-19} \theta,
$$

where the functional equation for $\zeta(s)$ was used to obtain the first term on the right.

Let

$$
s_{n}=\frac{1}{2}+i \gamma_{n}
$$

denote the $n$th zero of $\zeta(s)$ above the real axis. It is known that $\gamma_{1} \approx 14$ and $\gamma_{2} \approx 21$ (see Appendix); in particular, $\left|s_{1}\right|<\left|s_{2}\right|<22$ and thus

(10) $\zeta\left(2 s_{n}\right)=-\zeta\left(2-2 s_{n}\right) \frac{\Gamma\left(1-s_{n}\right)}{\Gamma\left(s_{n}\right)}\left(\frac{p}{4 \pi^{2}}\right)^{-i \gamma_{n}}+10^{-19} \theta, \quad(n=1,2)$.

Multiplying both sides by $\left(p / 4 \pi^{2}\right)^{i \gamma_{n}}\left(1 / \zeta\left(2 s_{n}\right)\right)$ and using the fact that $\left|\zeta\left(2 s_{n}\right)\right|>\frac{1}{2}$ for $n=1,2$ (see Appendix), we get

$$
\begin{aligned}
\left(\frac{p}{4 \pi^{2}}\right)^{i \gamma_{n}} & =-\frac{\zeta\left(2-2 s_{n}\right)}{\zeta\left(2 s_{n}\right)} \frac{\Gamma\left(1-s_{n}\right)}{\Gamma\left(s_{n}\right)}+2 \cdot 10^{-19} \theta \\
& =-\frac{\zeta\left(1-2 i \gamma_{n}\right) \Gamma\left(\frac{1}{2}-i \gamma_{n}\right)}{\zeta\left(1+2 i \gamma_{n}\right) \Gamma\left(\frac{1}{2}+i \gamma_{n}\right)}\left(1+2 \cdot 10^{-19} \theta\right), \quad(n=1,2) .
\end{aligned}
$$

Taking arguments of both sides of (11) gives

$$
\gamma_{n} \log \left(\frac{p}{4 \pi^{2}}\right)=a_{n}+2 \pi x_{n}+3 \cdot 10^{-19} \theta, \quad(n=1,2)
$$

where $x_{n}$ is an integer and

$$
\begin{aligned}
a_{n} & \equiv \pi-2 \arg \zeta\left(2 s_{n}\right)-2 \arg \Gamma\left(s_{n}\right) \quad(\bmod 2 \pi), \\
0 & \leqq a_{n}<2 \pi .
\end{aligned}
$$

Eliminating $\log \left(p / 4 \pi^{2}\right)$ from the equations (12), and solving for $x_{2}$, we obtain

$$
x_{2}=\frac{\gamma_{2}}{\gamma_{1}} x_{1}+a_{0}+10^{-18} \theta
$$

where

$$
a_{0}=\frac{1}{2 \pi}\left(\frac{\gamma_{2}}{\gamma_{1}} a_{1}-a_{2}\right)
$$

From the Appendix,

$$
\begin{aligned}
\frac{\gamma_{2}}{\gamma_{1}} & =1.487262003892890048+10^{-18} \theta, \\
a_{0} & =a+.4 \cdot 10^{-9} \theta \quad \text { where } a=-.461786352 .
\end{aligned}
$$


We can rewrite (14) as

$$
x_{2}=\frac{\gamma_{2}}{\gamma_{1}} x_{1}+a+\frac{1}{2} \cdot 10^{-9} \theta .
$$

Note that

$$
3.999999660=\frac{\gamma_{2}}{\gamma_{1}} \cdot 3+a+\frac{1}{2} \cdot 10^{-9} \theta .
$$

It is not accidental that $3\left(\gamma_{2} / \gamma_{1}\right)+a$ should be close to an integer; $x_{1}=3$ corresponds to $p=163$ (see introduction). In fact

$$
\begin{aligned}
& \gamma_{1} \log \left(\frac{163}{4 \pi^{2}}\right)=20.042984673072 \cdots \\
& a_{1}+2 \pi \cdot 3=20.042984673470 \cdots .
\end{aligned}
$$

(Compare this with (12), where these numbers would agree to at least 19 decimal places if $p>10,000$.) From (12), we now see that $p>10^{4}$ implies that $x_{1}>3$.

Subtracting (18) from (17) gives:

(19) $x_{2}-4=\frac{\gamma_{2}}{\gamma_{1}}\left(x_{1}-3\right)-b+10^{-9} \theta, \quad$ where $b=.000000340$.

Now let

$$
\begin{array}{ll}
p_{1}=83,532,765, & p_{2}=12,832,922, \\
q_{1}=56,165,467, & q_{2}=8,628,555 .
\end{array}
$$

Then $p_{1} q_{2}-q_{1} p_{2}=1$, so that $p_{1}$ and $q_{1}$ are relatively prime.

Also,

$$
\left|\left(\frac{\gamma_{2}}{\gamma_{1}}-\frac{p_{1}}{q_{1}}\right)\right|<2.3 \cdot 10^{-16}
$$

Let

$$
Q+\frac{R}{q_{1}}=\frac{p_{1}}{q_{1}}\left(x_{1}-3\right),
$$

where $0 \leqq R<q_{1}$ and $Q$ and $R$ are integers. Subtracting (22) from (19) giv es

$$
x_{2}-Q-4=\left(\frac{\gamma_{2}}{\gamma_{1}}-\frac{p_{1}}{q_{1}}\right)\left(x_{1}-3\right)+\left(\frac{R}{q_{1}}-b\right)+10^{-9} \theta .
$$

If $x_{1} \leqq 5.15 \cdot 10^{7}$, then

$$
\left|\left(\frac{\gamma_{2}}{\gamma_{1}}-\frac{p_{1}}{q_{1}}\right)\left(x_{1}-3\right)\right|<12 \cdot 10^{-9},
$$

and thus $x_{1} \leqq 5.1 \cdot 210^{7}$ implies 
(25)

$$
\left|x_{2}-Q-4\right|<12 \cdot 10^{-9}+\left(1-340 \cdot 10^{-9}\right)+10^{-9}<1 \text {. }
$$

On the other hand, since

$$
\frac{18}{q_{1}}<321 \cdot 10^{-9}<b=340 \cdot 10^{-9}<356 \cdot 10^{-9}<\frac{20}{q_{1}} \text {, }
$$

we find that $x_{1} \leqq 5.1 \cdot 10^{7}$ and $R \neq 19$ implies

$$
\begin{aligned}
\left|x_{2}-Q-4\right| & \geqq\left|\frac{R}{q_{1}}-b\right|-\left|\left(\frac{\gamma_{2}}{\gamma_{1}}-\frac{p_{1}}{q_{1}}\right)\left(x_{1}-3\right)\right|-10^{-9} \\
& >16 \cdot 10^{-9}-12 \cdot 10^{-9}-10^{-9}>0 .
\end{aligned}
$$

Inequalities (25) and (26) are contradictory, and therefore $x_{1} \leqq 5.1 \cdot 10^{7}$ implies $R=19$.

But if $R=19$, then we see from (22) that

and this implies

$$
19 \equiv p_{1}\left(x_{1}-3\right) \quad\left(\bmod q_{1}\right),
$$

$$
x_{1}-3 \equiv 51,611,611 \quad\left(\bmod q_{1}\right) .
$$

Thus under all circumstances, $x_{1}>5.1 \cdot 10^{7}$. Hence by (12),

$$
\begin{aligned}
\log \left(\frac{p}{4 \pi^{2}}\right) & =\frac{a_{1}+2 \pi x_{1}+3 \cdot 10^{-19} \theta}{\gamma_{1}} \\
& >\frac{2 \pi\left(5.1 \cdot 10^{7}\right)-3 \cdot 10^{-19}}{14.2} \\
& >2.2 \cdot 10^{7},
\end{aligned}
$$

and Theorem 1 follows.

\section{APPENDIX}

I wish to express my thanks to M.D. Bigg who furnished values of $\gamma_{1}$ and $\gamma_{2}$ to fifty decimal places and to R.S. Lehman who furnished values of $\arg \zeta\left(2 s_{n}\right)$ and $\left|\zeta\left(2 s_{n}\right)\right|$ for $n=1$ and 2 , with a proved accuracy of $\pm 10^{-10}$. The values of $\gamma_{1}$ and $\gamma_{2}$ were confirmed independently by Robert Spira to fifteen decimal places. Their values are:

(A1)

$$
\begin{aligned}
\gamma_{1} & =14.134725141734693790457+10^{-21} \theta, \\
\gamma_{2} & =21.022039638771554992628+10^{-21} \theta, \\
\frac{1}{\pi} \arg \zeta\left(2 s_{1}\right) & =-.108452737083095+10^{-10} \theta \quad(\bmod 2), \\
\frac{1}{\pi} \arg \zeta\left(2 s_{2}\right) & =.067103865503910+10^{-10} \theta \quad(\bmod 2), \\
\left|\zeta\left(2 s_{1}\right)\right|= & 1.94875731381740+10^{-10} \theta, \\
\left|\zeta\left(2 s_{2}\right)\right|= & .830962021546955+10^{-10} \theta .
\end{aligned}
$$


All other numbers were calculated on a Monroe desk calculator to fifteen places.

The Euler-Maclaurin sum formula for $\log \Gamma\left(\frac{1}{2}+i \gamma\right)$ can be expressed $([4, \mathrm{p} .132]$. It should be noted that the exponent $(m+1)$ in Nörlund's remainder term should be replaced by $m$. See also $[5$, p. 131]):

$$
\begin{aligned}
\log \Gamma\left(\frac{1}{2}+i \gamma\right)= & i \gamma \log (i \gamma)-i \gamma+\frac{1}{2} \log (2 \pi) \\
& +\sum_{j=2}^{11}(-1)^{j} \frac{B_{j}\left(\frac{1}{2}\right)}{j(j-1)} \cdot \frac{1}{(i \gamma)^{j-1}} \\
& -\int_{0}^{\infty} \frac{B_{11}\left(x-\frac{1}{2}-\left[x-\frac{1}{2}\right]\right)}{11(x+i \gamma)^{11}} d x
\end{aligned}
$$

From this we see that

$$
\begin{aligned}
& \arg \Gamma\left(\frac{1}{2}+i \gamma\right)=\gamma(\log \gamma-1)+\sum_{k=1}^{5} \frac{(-1)^{k} B_{2 k}\left(\frac{1}{2}\right)}{2 k(2 k-1) \gamma^{2 k-1}} \\
& -\operatorname{Im} \int_{0}^{\infty} \frac{B_{11}\left(x-\frac{1}{2}-\left[x-\frac{1}{2}\right]\right)}{11(x+i \gamma)^{11}} d x \text {. }
\end{aligned}
$$

From the formula $[5$, p. 123$]$,

we get the following:

$$
B_{k}\left(\frac{1}{2}\right)=-\left(1-2^{1-k}\right) B_{k},
$$

$$
\begin{aligned}
& \frac{B_{2}\left(\frac{1}{2}\right)}{2 \cdot \frac{1}{1}}=-.041666666666667+10^{-15} \theta \\
& \frac{B_{4}\left(\frac{1}{2}\right)}{4 \cdot 3}=.002430555555556+10^{-15} \theta \\
& \frac{B_{6}\left(\frac{1}{2}\right)}{6 \cdot 5}=-.000768849206349+10^{-15} \theta \\
& \frac{B_{8}\left(\frac{1}{2}\right)}{8 \cdot 7}=.000590587797619+10^{-15} \theta \\
& \frac{B_{10}\left(\frac{1}{2}\right)}{10 \cdot 9}=-.000840106797138+10^{-15} \theta
\end{aligned}
$$


From [6], we get

$$
\begin{aligned}
& \log \gamma_{1}=2.648634545730790+10^{-15} \theta, \\
& \log \gamma_{2}=3.045571393984561+10^{-15} \theta .
\end{aligned}
$$

Finally, for $t>14$, we get from (3),

$$
\begin{aligned}
\left|\int_{0}^{\infty} \frac{B_{11}\left(x-\frac{1}{2}-\left[x-\frac{1}{2}\right]\right)}{11(x+i t)^{11}} d x\right| & \leqq \frac{(2 \pi) \frac{2.11 !}{(2 \pi)^{11}} \cdot \frac{11}{10}}{11 t^{9}} \int_{0}^{\infty} \frac{d x}{x^{2}+t^{2}} \\
& =\frac{11 ! \pi}{10(2 \pi)^{11} t^{10}} \\
& <10^{-13} .
\end{aligned}
$$

Putting these numbers into (A2), we obtain

$$
\begin{aligned}
& \frac{1}{\pi} \arg \Gamma\left(s_{1}\right)=7.418512651985173+2 \cdot 10^{-13} \theta \\
& \frac{1}{\pi} \arg \Gamma\left(s_{2}\right)=13.688619111000235+2 \cdot 10^{-13} \theta .
\end{aligned}
$$

We may rewrite (12) thusly:

$$
\begin{aligned}
\frac{a_{n}}{2 \pi} \equiv \frac{1}{2}-\frac{1}{\pi} \arg \zeta\left(2 s_{n}\right)-\frac{1}{\pi} \arg \Gamma\left(s_{n}\right) & (\bmod 1), \\
& 0 \leqq \frac{a_{n}}{2 \pi}<1 .
\end{aligned}
$$

Using (A1) and (A3), we find that

$$
\begin{aligned}
& \frac{a_{1}}{12 \pi} \equiv .189940085097922+1.002 \cdot 10^{-10} \theta \quad(\bmod 1), \\
& \frac{a_{2}}{2 \pi} \equiv .744277023495855+1.002 \cdot 10^{-10} \theta \quad(\bmod 1) .
\end{aligned}
$$

Since these numbers are between 0 and 1 , the above is actually an equality. Finally,

$$
\frac{\gamma_{2}}{\gamma_{1}}=1.487262003892890048+10^{-18} \theta
$$

and

$$
\begin{aligned}
a_{0} & =\frac{\gamma_{2}}{\gamma_{1}}\left(\frac{a_{1}}{2 \pi}\right)-\frac{a_{2}}{2 \pi} \\
& =-.461786351913533+3 \cdot 10^{-10} \theta .
\end{aligned}
$$




\section{REFERENCES}

1. H. Heilbronn and E. H. Linfoot, On the imaginary quadratic corpora of class-number one, Quart. J. Math. Oxford Ser. 5 (1934), 293-301.

2. D. H. Lehmer, On imaginary quadratic fields whose class number is unity, Bull. Amer. Math. Soc. 39 (1933), 360.

3. C. Jordan, Calculus of finite differences, 2nd ed., Chelsea, New York, 1947.

4. N. E. Norlund, Mémoire sur le calcul aux differences Finies, Acta Math. 44 (1923), 71-212.

5. 5. J. F. Steffensen, Interpolation, 2nd ed., Chelsea, New York, 1950.

6. National Bureau of Standards, Table of natural logarithms for arguments between zero and five to sixteen decimal places, Applied Mathematics Series No. 31, U. S. Government Printing Office, Washington, D. C., 1953.

\section{UNIVERSITY OF MICHIGAN}

ANN ARbor, Michigan 